\title{
Residential wire codes: reproducibility and relation with measured magnetic fields
}

\author{
Robert E Tarone, William T Kaune, Martha S Linet, Elizabeth E Hatch, \\ Ruth A Kleinerman, Leslie L Robison, John D Boice Jr, Sholom Wacholder
}

Division of Cancer Epidemiology and Genetics, National Cancer Institute, Bethesda, Maryland, USA

R E Tarone

M S Linet

E E Hatch

R A Kleinerman

$\mathrm{J}$ D Boice Jr

$S$ Wacholder

EM Factors, Richland, Washington, USA

W T Kaune

Division of Pediatric Epidemiology and Clinical Research, University of Minnesota School of Medicine,

Minneapolis, Minnesota, USA

L L Robison

Correspondence to: Dr R Tarone, Division of Cancer Epidemiology and Genetics, National Cancer Institute, EPN 403, Bethesda, Maryland, USA 20892.

Accepted 31 October 1997

\begin{abstract}
Objectives-To investigate the reproducibility of wire codes to characterise residential power line configurations and to determine the extent to which wire codes provide a proxy measure of residential magnetic field strength in a casecontrol study of childhood leukaemia conducted in nine states within the United States.

Methods-Misclassification of wire codes was assessed with independent measurements by two technicians for 187 residences. The association between categories of wire code and measured level of magnetic field was evaluated in 858 residences with both a wire code measurement and a 24 hour measurement of the magnetic field in the bedroom. The strength of the association between category of wire code and risk of leukaemia was examined in two regions with different average levels of magnetic field in homes with high categories of wire code.

Results-The reproducibility of any of three different classifications of wire codes was excellent $(\kappa \geqslant 0.89)$. Mean and median magnetic fields, and the percentage of homes with high magnetic fields increased with increasing category for each of the wire code classification schemes. The size of the odds ratios for risk of leukaemia and high categories of wire code did not reflect the mean levels of the magnetic field in those categories in two study regions.

Conclusion-Misclassification of categories of wire code is not a major source of bias in the study. Wire codes provide a proxy measure of exposure to residential magnetic fields. If magnetic fields were a risk factor for leukaemia, however, there would be some attenuation of risk estimates based on wire codes because of misclassification of exposure to magnetic fields at both extremes of the wire code range. The lack of an association between high categories of wire code and risk of leukaemia cannot be explained by a failure of the wire code classification schemes to estimate exposure to magnetic fields in the study area.

(Occup Environ Med 1998;55:333-339)
\end{abstract}

Keywords: childhood acute lymphoblastic leukaemia; magnetic fields; exposure assessment; wire codes; misclassification
The investigation into a possible association between exposure to magnetic fields and cancer risk began with the report of a case-control study of childhood cancer in 1979. ${ }^{1}$ Exposure assessment in the Wertheimer and Leeper study was based not on systematic measurement of levels of magnetic field in study homes, but rather on a method for determining from visual inspection of nearby electrical power lines the potential for high current flow through power lines in the vicinity of study homes. This method of assessing exposure, which has been referred to as wire coding, categorises residences on the basis of the types and configurations of, and distances from, nearby electric power transmission and distribution lines. ${ }^{12}$ Wire coding has been used as a proxy measure of potential for residential exposure to magnetic fields induced by current flow in adjacent power lines in several subsequent epidemiological studies of childhood cancer. ${ }^{3-7}$

Average measurements of residential magnetic fields have been shown to increase with increasing category of wire code in Seattle, Denver, and Los Angeles. ${ }^{5-10}$ Some, ${ }^{145}$ but not all, $^{37}$ studies of childhood cancer have reported significant associations between cancer risk and wire codes. Significant associations have not been reported between direct magnetic field measurements and risk of childhood cancer, ${ }^{45711}$ which has raised questions about the interpretation of associations between wire code and cancer risk. ${ }^{12-17}$

The first wire coding system categorised homes as having either low current configuration or high current configuration. ${ }^{1}$ Wertheimer and Leeper expanded their wire coding scheme to include four categories-namely, very low current configuration (VLCC), ordinary low current configuration (OLCC), ordinary high current configuration (OHCC), and very high current configuration (VHCC). ${ }^{2} \mathrm{~A}$ category for homes with adjacent power lines buried underground (UG) was added later by other researchers. ${ }^{4}$ The Wertheimer-Leeper method assigns a wire code to a home on the basis of the shortest distance between the home and the nearest electrical power transmission line, three phase primary distribution line, first span secondary distribution line, short first span secondary distribution line, or second span secondary distribution line. Three phase primary distribution lines are further classified as thick or thin based on the diameter of their conductors.

In practice, it can be difficult to ascertain certain configurational variables required to 
Table 1 Distribution of 187 residences by initial and repeat quality control (QC) wire code category with the 5-level Wertheimer-Leeper classification

\begin{tabular}{llcccc}
\hline \multicolumn{5}{l}{ Repeat $Q C$ wire code } \\
\cline { 2 - 6 } & UG wire code & VLCC & OLCC & OHCC & VHCC \\
\hline UG & 7 & 0 & 0 & 0 & 0 \\
VLCC & 1 & 27 & 3 & 0 & 0 \\
OLCC & 0 & 0 & 31 & 4 & 0 \\
OHCC & 1 & 0 & 3 & 60 & 2 \\
VHCC & 0 & 1 & 0 & 0 & 47 \\
\hline
\end{tabular}

$\mathrm{UG}=$ underground wiring (no overhead powerlines within 150 feet of residence); VLCC=very low current configuration; $\mathrm{OLCC}=$ ordinary low current configuration; $\mathrm{OHCC}=$ ordinary high current configuration; VHCC=very high current configuration.

assign a wire code-for example, the distinction between different types of secondaries, and the conductor diameter assessed from a distance of 30 or more feet-leaving the results prone to error and misclassification. In an attempt to minimise such errors, Kaune and Savitz developed a simplified version of the Wertheimer-Leeper wire coding scheme which eliminated some of the more subjective and error prone elements of the original Wertheimer-Leeper system. ${ }^{18}$ The modified scheme results in a classification of residences into three categories, low, medium, or high. Savitz and Kaune ${ }^{6}$ applied the simplified system to data from an earlier study ${ }^{4}$ and obtained estimates of associations between childhood cancer and wire code that were similar in size to, but more precise than, those obtained with the Wertheimer-Leeper system.

A study of measured levels of magnetic fields and residential wire codes was initiated in 1989 by the National Cancer Institute (NCI) as part of a large, comprehensive case-control interview study of acute lymphoblastic leukaemia among children. ${ }^{19}{ }^{20}$ Enrolled in the study were childhood cases of acute lymphoblastic leukaemia receiving treatment from oncologists with the Children's Cancer Group (CCG) and population based controls selected by random digit dialing. A quality control component was incorporated into the wire code protocol to minimise, and measure the level of, misclassification. The results of this quality control study are described in the present report. The relation between categories of wire code and measured magnetic fields was evaluated to examine the extent to which wire codes provide a surrogate measure for contemporaneous residential exposure to electromagnetic fields in the region of the United States in which the NCI/CCG study was carried out. Also reported are the results of an examination of whether the size of the odds ratio (OR) for risk of acute lymphoblastic leukaemia and a high category of wire code depends upon the

Table 2 Distribution of 187 residences by initial and repeat quality control (QC) wire code category with the 3-level Kaune-Savitz classification

\begin{tabular}{lccc}
\hline \multirow{4}{*}{ Initial wire code } & \multicolumn{3}{l}{ Repeat $Q C$ wire code } \\
\cline { 2 - 4 } & Low & Medium & High \\
\hline Low & 49 & 1 & 0 \\
Medium & 1 & 53 & 2 \\
High & 2 & 3 & 76 \\
\hline
\end{tabular}

estimated difference in mean levels of magnetic fields between the high and reference wire codes.

\section{Materials and methods}

STUDY POPULATION

Eligibility criteria for subjects to be included in the wire code component of the case-control study have been described elsewhere. ${ }^{19}$ For a home of a case and that of the corresponding matched control to be eligible for wire coding, the case had to reside at the date of diagnosis, and the control to reside at the same date, in one of nine states: Wisconsin; Iowa; Minnesota; Illinois; Indiana; Michigan; Ohio; Pennsylvania; and New Jersey. The date of diagnosis of leukaemia is referred to as the reference date for both the case and its matched control. The present report includes all residences that were assigned a wire code, including some residences of children for whom the corresponding controls or cases were subsequently determined not to be eligible for wire coding, and residences of potential controls who refused to participate in the case-control study, but whose homes were still wire coded to identify potential differences in the distribution of wire codes between refusers and participants. Wire coding of the homes of children whose parents refused to participate was possible because it was usually not necessary to have access to the property on which a residence is situated to assign a wire code.

POWER LINE DIAGRAMMING AND ASSIGNMENT OF WIRE CODES

Two technicians were trained over a five day period by one of us (WTK) in a standardised approach for visually inspecting and diagramming overhead power lines within 150 feet of a residence. Shortly after initiation of data collection for the case-control study, the two technicians and the trainer independently diagrammed 19 homes with good reproducibility. The same two technicians diagrammed study homes during three to four month periods in each of the four years of data collection. The technicians were retrained before each year's wire coding endeavours. Wire code technicians were blinded to the case-control status of the subject living in each residence, but occasionally learned the status from residents while in the course of their inspection. A computerised algorithm assigned a category of wire code to each residence based on data abstracted from wiring diagrams. The wire code depends on the type and configuration of power lines, and the shortest distance between the power line and the exterior of the residence.

WIRE CODE QUALITY CONTROL PROTOCOL In each year of data collection a $10 \%$ randomly generated sample of eligible residences was selected for quality control study. Each of these homes was inspected and diagrammed independently by both technicians. Also, about $10 \%$ of residences wire coded in each of the first three years of data collection were selected to be re-examined the next year. Each residence in this sample was inspected and 
Table 3 Summary of 24 hour magnetic field measurements in the bedrooms of residences by wire code category using the 5-level Wertheimer-Leeper wire code classification

\begin{tabular}{llllll}
\hline & \multicolumn{5}{l}{ Wire code category } \\
\cline { 2 - 6 } & UG & VLCC & OLCC & OHCC & VHCC \\
\hline Homes $(\mathrm{n})$ & 150 & 221 & 262 & 170 & 55 \\
Mean $(\mu \mathrm{T})$ & 0.064 & 0.077 & 0.118 & 0.136 & 0.207 \\
Median $(\mu \mathrm{T})$ & 0.046 & 0.049 & 0.075 & 0.098 & 0.133 \\
Field $>0.2 \mu \mathrm{T}^{\star}$ & $4(3)$ & $14(6)$ & $38(15)$ & $34(20)$ & $22(40)$ \\
Field $>0.3 \mu \mathrm{T}^{\star}$ & $0(0)$ & $7(3)$ & $16(6)$ & $12(10)$ & $12(22)$ \\
\hline
\end{tabular}

${ }^{\star}$ Homes (n (\%)) with measurement exceeding specified level.

Other footnotes as for table 1 .

diagrammed by the technician who had not evaluated the home the previous year. This sample included all homes assigned to the highest (VHCC) category in the initial visit, as well as homes initially classified in the OHCC category that were deemed most likely to be homes that should have been classified as VHCC, but were misclassified as OHCC. Included in this group were homes initially determined to be $25-50$ feet from thin three phase primary lines, because these homes would have been classified as VHCC if the primary had actually been determined to be thick or if the distance had been estimated to be less than 25 feet. This group also included homes diagrammed as being 50-55 feet from thick three phase primary lines, because these homes would have been classified VHCC if the distance had actually been determined to be 50 feet or less. In total, there were 187 residences in the quality control sample.

MEASUREMENTS OF MAGNETIC FIELDS

Trained data collectors measured levels of magnetic fields with an Emdex-C meter (Electric Field Measurements Company, West Stockbridge, MA, USA) with a standard protocol. The Emdex-C is a programmable magnetic field meter that uses a three axis induction coil sensor. The bandwidth of an Emdex-C meter extends from about 40-300 $\mathrm{Hz}$. The dynamic range of the meter extends from $0.01-100 \mu \mathrm{T}$ at least, the upper limit being far in excess of field levels actually measured in any home evaluated in the NCI/CCG study. Internal noise in the meter limits its accuracy for fields $<0.03 \mu \mathrm{T}$.

The primary magnetic field measurement was a 24 hour recording taken in the bedroom of the study subject. The meter was programmed to take two measurements separated by one second, and then to wait 29 seconds before repeating the cycle. The meter was placed in a sealed box usually located under the

Table 4 Summary of 24 hour magnetic field measurements in the bedrooms of residences by wire code category using the 3-level Kaune-Savitz wire code classification

\begin{tabular}{llll}
\hline & \multicolumn{3}{l}{ Wire code category } \\
\cline { 2 - 4 } & Low & Medium & High \\
\hline Homes $(\mathrm{n})$ & 517 & 235 & 106 \\
Mean $(\mu \mathrm{T})$ & 0.082 & 0.124 & 0.193 \\
Median $(\mu \mathrm{T})$ & 0.052 & 0.087 & 0.132 \\
Field $>0.2 \mu \mathrm{T}^{\star}$ & $34(7)$ & $40(17)$ & $38(36)$ \\
Field $>0.3 \mu \mathrm{T}^{\star}$ & $14(3)$ & $17(7)$ & $21(20)$ \\
\hline
\end{tabular}

${ }^{\star}$ Homes (n (\%)) with measurement exceeding specified level. subject's bed at a place where the magnetic field did not differ by more than $20 \%$ from the field on the top surface of the bed at the usual location of the subject's torso while sleeping. If the meter could not be placed under the bed it was placed beside the bed at a location that satisfied the field test criteria described above. In residences no longer occupied by subjects at the time of the measurements, or where the subject's bed had been moved since the reference date, the meter was placed where the bed had been located during the year preceding the reference date, or, if the subject had moved before the reference date, where the bed had been during the 12 month period before moving. Spot magnetic field measurements of short duration $(30 \mathrm{~s})$ were also taken at various places in each residence with a hand held Emdex-C meter programmed to record the magnetic field at one second intervals. Because such spot measurements may have depended on the time of day they were taken, the 24 hour bedroom measurements were used to assess the relation between category of wire code and measured magnetic field. Analyses were based on the mean of all measurements taken over the 24 hour period in each residence. The 1258 subjects in the NCI/CCG study lived in 1610 homes that were eligible for measurements of magnetic fields, and a 24 hour measurement in the bedroom was obtained in 1360 of the eligible homes. ${ }^{20}$ Wire coding was restricted to homes lived in for at least $70 \%$ of the five year period before the reference date, and thus the evaluation of the relation between wire code and measured magnetic field was based on the 858 homes with both a wire code and a 24 hour measurement in the bedroom.

STATISTICAL ANALYSIS

The degree of agreement between the assigned wire codes with data from diagrams drawn by the two technicians was assessed with the $\kappa$ coefficient $^{21} ; 95 \%$ confidence intervals $(95 \%$ CIs) for $\kappa$ were calculated with the method of Fleiss et $a .^{22}$ Previous studies of childhood cancer and directly measured residential magnetic fields used as their primary measure of association the OR for subjects in residences with measurements of magnetic fields greater than a selected cut off point, usually $\geqslant 0.2 \mu \mathrm{T}$ $(2.0 \mathrm{mG})$. Thus, although mean and median magnetic fields are presented by the wire code, detailed analysis of the relation between wire code and measured magnetic fields was based on the examination of the proportion of homes in each wire code with measured magnetic field $>0.2$ or $>0.3 \mu \mathrm{T}$. All statistical tests were performed with data stratified by state. Exact methods were used to compare proportions in adjacent categories, ${ }^{23}$ and tests for trend in the proportions with increasing wire codes were performed with approximate tests. ${ }^{24}$ Two sided $\mathrm{p}$ values are reported.

Matched pairs analyses were used to evaluate the risk of acute lymphoblastic leukaemia relative to Wertheimer-Leeper categories of wire code. The UG and VLCC categories were combined to form the control category. ${ }^{17}$ Conditional logistic regression was used to control 
Table 5 Mean 24 hour magnetic field bedroom measurement $(\mu T)$ by Wertheimer-Leeper wire code category and by state

\begin{tabular}{llllll}
\hline \multicolumn{7}{l}{ Wire code category } \\
\cline { 2 - 6 } State & $\begin{array}{l}\text { UG } \\
\mu T(n) t\end{array}$ & $\begin{array}{l}\text { VLCC } \\
\mu T(n)\end{array}$ & $\begin{array}{l}\text { OLCC } \\
\mu T(n)\end{array}$ & $\begin{array}{l}\text { OHCC } \\
\mu T(n)\end{array}$ & $\begin{array}{l}\text { VHCC } \\
\mu T(n)\end{array}$ \\
\hline Illinois & $0.061(14)$ & $0.068(15)$ & $0.090(32)$ & $0.148(23)$ & $0.267(6)$ \\
Iowa & $0.090(8)$ & $0.074(29)$ & $0.140(20)$ & $0.101(9)$ & $0.219(7)$ \\
Indiana & $0.123(2)$ & $0.088(23)$ & $0.152(19)$ & $0.127(7)$ & $0.323(3)$ \\
Michigan & $0.072(19)$ & $0.051(30)$ & $0.108(45)$ & $0.168(23)$ & $0.102(4)$ \\
Minnesota & $0.071(24)$ & $0.090(27)$ & $0.151(30)$ & $0.148(13)$ & $0.082(3)$ \\
New Jersey & $0.056(20)$ & $0.084(16)$ & $0.073(26)$ & $0.087(22)$ & $0.218(9)$ \\
Ohio & $0.063(31)$ & $0.083(37)$ & $0.109(48)$ & $0.142(22)$ & $0.223(9)$ \\
Pennsylvania & $0.053(31)$ & $0.086(39)$ & $0.138(41)$ & $0.142(52)$ & $0.190(14)$ \\
\hline
\end{tabular}

* There were too few Wisconsin homes to provide useful information.

†Number of homes in parentheses.

Other footnotes as for table 1 .

for sex, mother's educational level, and family income.

\section{Results}

Wire code assessments were repeated for 187 residences. By design, the quality control sample included a disproportionate number of VHCC and OHCC residences; $26 \%$ and $35 \%$ in the quality control sample were VHCC and OHCC compared with $7 \%$ and $21 \%$ of all residences wire coded in the study, respectively. Table 1 gives the distribution of the 187 residences by initial and repeat quality control wire code classification with the five-level Wertheimer-Leeper wire code (including the UG category for underground wiring). The high level of agreement between the two technicians is evident, with complete agreement on $172(92 \%)$ of the residences. The $\kappa$ coefficient was 0.89 (95\% CI 0.84 to 0.94$)$ for the five-level code and 0.90 (0.85 to 0.95$)$ for the four-level code (with the UG and VLCC categories combined). Of the 15 residences where the technicians failed to agree on the five-level code, seven were due to different determinations of the distance from the residence to a power line, one to different assessments of the thickness of the primary distribution line conductor, and seven to differences in the identification of the type of power line-for example, primary or secondary distribution line. Table 2 compares the KauneSavitz wire codes assigned from the initial and repeat diagrams for the 187 residences in the quality control sample. With the modified code there was complete agreement on $178(95 \%)$ of residences, and the $\kappa$ coefficient was $0.93(0.88$ to 0.97 ). Of the nine residences where the technicians disagreed, four were due to differ-

Table 6 Risk of childhood acute lymphoblastic leukaemia according to Wertheimer-Leeper wire code classification of main residence ${ }^{\star}$

\begin{tabular}{|c|c|c|c|c|c|c|}
\hline \multirow[b]{2}{*}{ Wire code } & \multicolumn{3}{|c|}{ Michigan/Minnesota } & \multicolumn{3}{|c|}{ Other Statest } \\
\hline & Cases & Controls & OR $(95 \% C I) \neq$ & Cases & Controls & OR $(95 \% C I)$ \\
\hline UG+VLCC & 41 & 40 & 1.00 & 133 & 134 & 1.00 \\
\hline OLCC & 31 & 25 & $1.10(0.50$ to 2.43$)$ & 84 & 89 & 1.01 (0.66to 1.54$)$ \\
\hline OHCC & 15 & 23 & $0.60(0.25$ to 1.44$)$ & 72 & 63 & $1.17(0.73$ to 1.86$)$ \\
\hline VHCC & 2 & 1 & $2.46(0.18$ to 33.4$)$ & 22 & 25 & $0.81(0.42$ to 1.54$)$ \\
\hline
\end{tabular}

${ }^{\star}$ The number of matched pairs is two less than in the original report, ${ }^{20}$ because two pairs in which one member resided in Michigan or Minnesota and the other member resided in one of the other states in the study have been excluded.

tIllinois, Indiana, Iowa, New Jersey, Ohio, Pennsylvania, and Wisconsin.

$\ddagger$ Odds ratios are adjusted with conditional logistic regression for sex, mother’s educational level, and family income. ent measurements of distances from power lines to residences, and five to differences in classification of the type of power line.

Of the 858 residences in the study with both a wire code and a 24 hour magnetic field measurement in the bedroom, $150(17 \%)$ were in the UG category, $221(26 \%)$ were VLCC, $262(31 \%)$ were OLCC, $170(20 \%)$ were OHCC, and $55(6 \%)$ were VHCC. Table 3 shows the means and medians of the 24 hour measurements in the bedroom by five-level Wertheimer-Leeper wire code. As in previous studies, there was little difference between UG and VLCC categories in the levels of magnetic field measured, but both mean and median levels of magnetic fields clearly increased as the category of wire code rose from UG to VHCC. ${ }^{17}$ Table 3 shows the percentages of residences with 24 hour measurements in the bedroom $>0.2 \mu \mathrm{T}$ or $>0.3 \mu \mathrm{T}$ by wire code category. For values $>0.2 \mu \mathrm{T}$, the percentages rise with increasing category of wire code $\left(\mathrm{p}<10^{-6}\right)$, with significant increases between VLCC and OLCC categories $(p=0.0015)$ and between OHCC and VHCC categories $(\mathrm{p}=0.0034)$. For values $>0.3 \mu \mathrm{T}$, the percentages rise with increasing wire code $\left(\mathrm{p}<10^{-6}\right)$, with a significant increase between OHCC and VHCC categories $(p=0.033)$.

Classification of the 858 homes with the Kaune-Savitz wire code placed $517(60 \%)$ residences in the low category, $235(27 \%)$ residences in the medium category, and $106(12 \%)$ residences in the high category. Table 4 shows the mean and median of the 24 hour measurements in the bedroom by the modified wire code, and both measures show a clear increase in measured magnetic fields with increasing wire code. Table 4 also shows the percentages of residences with 24 hour measurements in the bedroom $>0.2 \mu \mathrm{T}$ and $>0.3 \mu \mathrm{T}$. For values $>0.2 \mu \mathrm{T}$ and $>0.3 \mu \mathrm{T}$, the increases are significant both for medium compared with low category homes $\left(\mathrm{p}<10^{-4}\right.$ and $\mathrm{p}=0.004$, respectively) and for high compared with medium category homes $(p=0.0004$ and $p=0.001$, respectively); the trend test was highly significant $\left(\mathrm{p}<10^{-6}\right)$ for both $>0.2$ and $>0.3 \mu \mathrm{T}$.

It has been suggested that the NCI/CCG study failed to find an association between categories of wire code and childhood risk of acute lymphoblastic leukaemia because wire codes are not as predictive of measured magnetic fields in the NCI/CCG study area as they were in previous study areas (Wertheimer N, Leeper E, Microwave News, July/August 1997, pp 12-14). To examine site specific variation in the predictive ability of wire codes, table 5 shows the mean 24 hour measurement in the bedroom by Wertheimer-Leeper categories and by state. Data from all states showed a tendency for measured fields to increase with increasing wire codes, but in Michigan and Minnesota the average level of magnetic fields in VHCC homes (four in Michigan and three in Minnesota) was lower than the average level of magnetic fields in both OHCC and OLCC homes. Table 6 shows the ORs for the association between risk of acute lymphoblastic leukaemia and categories of wire code for 
Table 7 Median 24 hour magnetic field bedroom measurement $(\mu T)$ by the Wertheimer-Leeper and the Kaune-Savitz modified wire code categories

\begin{tabular}{|c|c|c|c|c|c|}
\hline \multirow[b]{2}{*}{$\begin{array}{l}\text { Modified wire } \\
\text { code }\end{array}$} & \multicolumn{5}{|c|}{ Wertheimer-Leeper wire code } \\
\hline & $\begin{array}{l}U G \\
\mu T(n)^{*}\end{array}$ & $\begin{array}{l}V L C C \\
\mu T(n)\end{array}$ & $\begin{array}{l}O L C C \\
\mu T(n)\end{array}$ & $\begin{array}{l}\text { OHCC } \\
\mu T(n)\end{array}$ & $\begin{array}{l}V H C C \\
\mu T(n)\end{array}$ \\
\hline Low & $0.046(150)$ & $0.049(214)$ & $0.061(134)$ & $0.070(19)$ & $-(0)$ \\
\hline Medium & $-(0)$ & $0.097(7)$ & $0.085(128)$ & $0.093(100)$ & $-(0)$ \\
\hline High & $-(0)$ & $-(0)$ & $-(0)$ & $0.124(51)$ & $0.133(55)$ \\
\hline
\end{tabular}

^Number of homes.

Other footnotes as for table 1 .
Minnesota and Michigan combined, and for the remaining states combined. The OR for VHCC homes in the states for which VHCC homes had the highest average magnetic fields was 0.81 (95\% CI, 0.42 to 1.54$)$, compared with 0.88 (0.48 to 1.63$)$ for the entire study population. ${ }^{20}$ Thus the association was not strengthened by removing the states in which VHCC homes had low measured fields. Similarly, although Michigan and Minnesota had the highest measured fields in OHCC homes among the states in the study, the OR for the OHCC category in these two states was 0.60 (0.25 to 1.44$)$ compared with 1.17 (0.73 to 1.86 ) in the other states.

Table 7 shows a cross tabulation of the fivelevel Wertheimer-Leeper and the three-level Kaune-Savitz wire code classifications for the 858 residences. This table also includes the medians of the 24 hour measurements in the bedroom cross classified by both wire coding schemes. For residences classified in the low category with the Kaune-Savitz scheme there is some increase in measured fields for residences classified as OLCC and OHCC according to the Wertheimer-Leeper scheme compared with those classified as UG and VLCC, but there is no increase in measured field within the medium Kaune-Savitz category in going from VLCC to OHCC, and little difference within the high Kaune-Savitz category between residences classified as VHCC compared with those designated as OHCC. By contrast, within each level of the Wertheimer-Leeper wire code classification there is a clear increase in measured magnetic field with increasing category of the Kaune-Savitz wire code.

\section{Discussion}

Agreement between the wire codes assigned with data from duplicate diagrams drawn by the two technicians was exceptional. Values of $\kappa$ were close to one, despite the enrichment of the quality control sample with residences which were considered most likely to be misclassified. It is apparent that wire code misclassification could not have been a major source of bias in our case-control investigation of a possible association between high wire codes and increased risk of acute lymphoblastic leukaemia.${ }^{19}$ The excellent agreement between technicians is due, in part, to the comprehensive initial training and subsequent yearly retraining in the standardised approach to inspecting and diagramming power lines. The high level of agreement between wire code technicians was also enhanced by having highly experienced technicians who remained with the study for the entire four years.

The highly significant increase in mean 24 hour levels of magnetic field in the bedroom with increasing wire codes in the NCI/CCG study area indicates that wire code classifications can serve as surrogates for contemporaneous magnetic field measurement in the NCI/ CCG study. Some misclassification of bedroom measurements by wire code is inevitable, if only because the distance between the relevant power line and the measured bedroom can differ considerably from the distance that determines the wire code for that home (the shortest distance between the power line and the nearest point on an exterior wall). It is apparent that the use of wire codes as a surrogate for exposure to magnetic fields will, therefore, lead to an attenuated estimate of relative risk if high levels of magnetic fields are associated with an increased risk of disease. For example, with the Wertheimer-Leeper code, $60 \%$ of the VHCC residences had measured fields $<0.2 \mu \mathrm{T}$, whereas $5 \%$ of the very low UG and VLCC residences had measured fields $>0.2 \mu \mathrm{T}$. Similarly, when the Kaune-Savitz code was used, $64 \%$ of high category residences had measured fields $<0.2 \mu \mathrm{T}$, whereas $7 \%$ of low category residences had measured fields $>0.2 \mu \mathrm{T}$. Thus, although the ORs based on the comparison of the highest wire codes with the lowest category should exceed 1.0 for both classification schemes if measured fields $>0.2 \mu \mathrm{T}$ are associated with increased risk of disease, the magnitude of the ORs would be reduced by misclassification on both extremes of the wire code range.

The ORs for VHCC residences in the Denver study and the Los Angeles study were similar, despite substantial differences in the average levels of magnetic field measured in VHCC homes (and in the VHCC homes $v$ UG or LHCC homes) between the two cities. The percentage of VHCC homes of children in Denver with average spot levels $>0.2 \mu \mathrm{T}$ was $60 \%$ and the median level in VHCC homes was $0.216 \mu \mathrm{T} .{ }^{4}$ Although this is considerably higher than the median 24 hour level of $0.133 \mu \mathrm{T}$ in bedrooms for VHCC homes in the NCI/CCG study, the Denver median is based on only 12 homes ( $v 55$ in the NCI/CCG study), and the corresponding mean of $0.212 \mu \mathrm{T}$ for Denver VHCC homes is similar to the mean of 0.207 $\mu \mathrm{T}$ in the NCI/CCG study. In the Los Angeles childhood leukaemia study, the geometric mean of 24 hour average levels in bedrooms of VHCC homes was $0.115 \mu \mathrm{T},{ }^{5}$ and in a Los Angeles childhood brain cancer study, 35\% of VHCC homes had 24 hour average levels in bedrooms $>0.2 \mu \mathrm{T}$, and the median of 24 hour average bedroom levels in VHCC homes was $0.108 \mu \mathrm{T} .{ }^{11}$ Intermediate values were obtained for the NCI/CCG study, in which $40 \%$ of VHCC homes had 24 hour average levels in bedrooms $>0.2 \mu \mathrm{T}$ and the median was 0.133 $\mu \mathrm{T}$ for VHCC homes. Thus any difference between the NCI/CCG study and the Denver and Los Angeles studies in inferences for homes with high wire codes are unlikely to be explained by differences among the three study 
areas in the relation between wire code and measured magnetic fields.

Exclusion of data from two states with low average measured magnetic fields in VHCC homes (Michigan and Minnesota) led to a reduction in the $\mathrm{OR}$ for the association between childhood acute lymphoblastic leukaemia and VHCC homes from 0.88 to 0.81 . Furthermore, Michigan and Minnesota had the highest average measured magnetic fields in OHCC homes among states in the study, but the OR for OHCC homes in these two states was 0.60 compared with 1.17 in the other states. Although data from the NCI/CCG study suggest that wire code is as good a surrogate for measured magnetic fields as in earlier studies, even if the association between wire code and measured field were somewhat weaker than in earlier studies, odds ratios would still not be expected to be $<1$ for high wire code if magnetic fields are, indeed, a risk factor for childhood acute lymphoblastic leukaemia. The lack of a positive association between wire code and risk of leukaemia in the NCI/CCG study cannot, then, be explained in terms of the strength of the association between wire code category and measured field level.

The evaluation of residential magnetic field measurements cross classified by both Wertheimer-Leeper and Kaune-Savitz wire codes (table 7) showed that the Kaune-Savitz code provided additional discrimination (for each category of the Wertheimer-Leeper code, median levels for magnetic fields rose with increasing category of the Kaune-Savitz code), although the Wertheimer-Leeper classification provides little additional discrimination within each category of the Kaune-Savitz code. In addition, the Kaune-Savitz code resulted in almost twice as many homes being assigned to the highest category compared with the Wertheimer-Leeper code, without an appreciable decrease in measured magnetic fields in homes in the highest category (median 0.132 $\mu \mathrm{T}$ for homes in the Kaune-Savitz high category $v 0.133 \mu \mathrm{T}$ for homes in the Wertheimer-Leeper VHCC category).

Although the modified code seems in some ways to be a better predictor of exposure to magnetic fields, comparison of the magnetic field measurements in the highest and lowest categories (tables 3 and 4) suggests that the difference in magnetic field measurements between extreme wire code categories is larger for the Wertheimer-Leeper classifications (with or without an UG category) than for the Kaune-Savitz wire code classification. The difference is small in the NCI/CCG study area, but may be larger in other regions. It is unclear, in general, which coding system will provide more statistical power when comparing the highest category with the lowest. The larger number of residences in extreme wire code categories favours the Kaune-Savitz code (the numbers of residences in the high and low categories will generally be larger than the corresponding numbers in the VHCC and UG or combined UG and VLCC categories, respectively). Consideration of the size of the difference between measurements of magnetic field in extreme categories, on the other hand, may favour the four-level or five-level Wertheimer-Leeper wire code. Accordingly, exposure assessment with the Kaune-Savitz code could produce ORs that are smaller, but more precise, than those based on the Wertheimer-Leeper code. ${ }^{6}$

Wire code classifications seem to provide a useful surrogate for potential exposure to magnetic fields in the mid-western and midAtlantic study area in the United States. Contemporaneous magnetic field measurements are also proxy measurements for past exposure to magnetic fields during the (as yet unknown) aetiologically relevant period for childhood acute lymphoblastic leukaemia, and it is unclear which proxy measure is best for epidemiological investigations. ${ }^{14-17}$ As already noted, there is misclassification by wire codes of contemporaneous measured magnetic fields. It seems indisputable, therefore, that the use of wire codes as a proxy measure of levels of magnetic fields will also misclassify exposures before diagnosis that are of primary interest in assessing risk of leukaemia. Consequently, past findings of significant associations between childhood leukaemia and wire codes, without comparable associations for measured fields, would seem to indicate that either (a) wire codes are better predictors of average historic exposure to magnetic fields than are contemporaneous measurements of magnetic fields, ${ }^{14}{ }^{15}{ }^{17}(b)$ wire codes are better predictors of some aetiologically important aspect of magnetic fields than are average measurements of magnetic fields, ${ }^{17}$ (c) wire codes are a surrogate for some risk factor unrelated to power frequency magnetic fields, ${ }^{16}$ or $(d)$ the positive associations between wire codes and disease were due to bias. The NCI/CCG case-control study of childhood leukaemia allows a more informative comparison of associations between disease and wire codes and associations between disease and measured magnetic fields than previous studies, because magnetic field measurements were taken closer to the time of diagnosis than was possible in previous studies, data were collected on many possible confounding variables, and restrictive residency criteria leading to possible control selection bias in some previous studies were avoided. ${ }^{19}$

The NCI/CCG study found that risk of childhood acute lymphoblastic leukaemia decreased non-significantly with increasing Wertheimer-Leeper wire code category, but increased non-significantly with increasing measurements of magnetic field strength. Because the controversy surrounding magnetic fields and risk of leukaemia has been driven almost entirely by findings with wire codes or other surrogates for exposure, ${ }^{14525}$ the lack of an association between wire codes and leukaemia in the NCI/CCG study is noteworthy. Living near electrical power transmission and distribution lines did not increase the risk of childhood leukaemia. ${ }^{20}$ Divergence in the direction of the trends in risk for measured magnetic fields and visual wire codes, given the positive association between wire codes and 
measurements, would be a paradox only if residential magnetic fields are a risk factor for childhood leukaemia. Results of the NCI/CCG study provide little support for this hypothesis. ${ }^{20}$

Fieldwork was performed under a contract from the National Cancer Institute, N01-CP-95608 to Westat, Rockville, Maryland. We thank Carol Haines of Westat, Rockville, Maryland, for land. We thank Carol Haines of Westat, Rockville, Maryland, for directing field work, Bob and Esther Workley of Enertech Consultants, Campbell, California, for performing the wire coding and Shelley Niwa of Westat and Charlene Hartsock of Information Management Services, Rockville, Maryland, for computer support.

1 Wertheimer N, Leeper E. Electrical wiring configuration and childhood cancer. Am 7 Epidemiol 1979;109:273-84.

2 and childhood cancer. Am f Epidemiol 1979;109:273-84. wires near the home. Int f Epidemiol 1982;11:345-55.

3 Fulton JP, Cobb S, Peble L, et al. Electrical wiring configurations and childhood leukemia in Rhode Island. Am f Epidemiol 1980;111:292-6.

4 Savitz DA, Wachtel H, Barnes FA, et al. Case-control study of childhood cancer and exposure to 60-hertz magnetic fields. Am f Epidemiol 1988;128:21-38

5 London SJ, Thomas DC, Bowman JD, et al. Exposure to residential electric and magnetic fields and risk of childhood leukemia. Am f Epidemiol 1991;134:923-37.

6 Savitz DA, Kaune WT. Childhood cancer in relation to a modified residential wire code. Environ Health Perspect 1993;101:76-80.

7 Gurney JG, Mueller BA, Davis S, et al. Childhood brain tumor occurrence in relation to residential power line configurations, electric heating sources, and electric appliance use. Am ₹ Epidemiol 1996;143:120-8.

8 Kaune WT, Stevens RG, Callahan NJ, et al. Residential magnetic and electric fields. Bioelectromagnetics 1987;8: 315-35.

9 Barnes F, Wachtel H, Savitz D, et al. Use of wiring configuration and wiring codes for estimating externally generated electric and magnetic fields. Bioelectromagnetics 1989;10: 13-21.

10 Dovan T, Kaune WT, Savitz D. Repeatability of measurements of residential magnetic fields and wire codes. Bioelectromagnetics 1993;14:145-59.
11 Preston-Martin S, Navidi W, Thomas D, et al. Los Angeles study of residential magnetic fields and childhood brain tumors. Am ₹ Epidemiol 1996;143:105-19.

12 Jones TL, Shih CH, Thurston DH, et al. Selection bias from differential residential mobility as an explanation for associations of wire codes with childhood cancer. 7 Clin Epidemiol 1993;46:545-8.

13 Gurney JG, Davis S, Schwarz SM, et al. Childhood cancer occurrence in relation to power line configurations: a study of potential selection bias in case-control studies. Epidemiology 1995;6:31-5.

14 Feychting M, Kaune WT, Savitz DA, et al. Estimating exposure in studies of residential magnetic fields and cancer: importance of short-term variability, time interval between diagnosis and measurement, and distance to power line. Epidemiology 1996;7:220-4.

15 Neutra RR, del Pizzo V. When "wire codes" predict cancer better than spot measurements of magnetic fields. Epidemiology 1996;7:217-8.

16 Committee on the Possible Effects of Electromagnetic Fields on Biological Systems. Possible health effects of exposure to residential electric and magnetic fields. Washington, DC: National Academy Press, 1996.

17 Kheifets LI, Kavet R, Sussman SS. Wire codes, magnetic fields, and childhood cancer. Bioelectromagnetics 1997;18: 99-110

18 Kaune WT, Savitz DA. Simplification of the WertheimerLeeper wire code. Bioelectromagnetics 1994;15:275-82.

19 Kleinerman RA, Linet MS, Hatch EE, et al. Magnetic field exposure assessment in a case-control study of childhood leukemia. Epidemiology 1997;8:575-83.

20 Linet MS, Hatch EE, Kleinerman RA, et al. Residential exposure to magnetic fields and acute lymphoblastic leukemia in children. N Engl f Med 1997;337:1-7.

21 Cohen J. A coefficient of agreement for nominal scales. Educational Psychological Measurement 1960;20:37-46.

22 Fleiss JL, Cohen J, Everitt BS. Large-sample standard errors of kappa and weighted kappa. Psychol Bull 1969;72:323-7.

23 Thomas DG, Gart JJ. Improved and extended exact and asymptotic methods for the combination of $2 \times 2$ tables. Comput Biomed Res 1992;25:75-84.

24 Mantel N. Chi-square tests with one degree of freedom; extensions of the Mantel-Haenszel procedure. fournal of the American Statistical Association 1963;58:690-700.

25 Feychting M, Ahlbom A. Magnetic fields and cancer in children residing near Swedish high-voltage power lines. Am $\mathcal{F}$ Epidemiol 1993;138:467-81.

\section{Occupational and Environmental Medicine and the electronic age}

OEM has an Email address which is 100632.3615@compuserve.com. We welcome contact by Email, including letters to the editor. Many of our reviewers send us their reports by Email, helping to speed up the peer review process.
Our publishing system is now fully electronic, and authors are sending their revised copy to us on disk as well as paper. Watch for revised Instructions to Authors.

The Editor 